\title{
Geometrically Correct 3-D Reconstruction of Intravascular Ultrasound Images by Fusion with Biplane Angiography - Methods and Validation
}

\author{
Andreas Wahle, ${ }^{*}$ Member, IEEE, Guido P. M. Prause, Steven C. DeJong, and Milan Sonka, Member, IEEE
}

\begin{abstract}
In the rapidly evolving field of intravascular ultrasound (IVUS), the assessment of vessel morphology still lacks a geometrically correct 3-D reconstruction. The IVUS frames are usually stacked up to form a straight vessel, neglecting curvature and the axial twisting of the catheter during the pullback. Our method combines the information about vessel cross-sections obtained from IVUS with the information about the vessel geometry derived from biplane angiography. First, the catheter path is reconstructed from its biplane projections, resulting in a spatial model. The locations of the IVUS frames are determined and their orientations relative to each other are calculated using a discrete approximation of the Frenet-Serret formulas known from differential geometry. The absolute orientation of the frame set is established utilizing the imaging catheter itself as an artificial landmark. The IVUS images are segmented using our previously developed algorithm. The fusion approach has been extensively validated in computer simulations, phantoms, and cadaveric pig hearts.

Keywords-Coronary Artery System, Intravascular Ultrasound, Biplane Angiography, Multimodal Image Fusion.
\end{abstract}

\section{INTRODUCTION}

A CCURATE ASSESSMENT of vessel lesions like stenoses or diffuse alterations is indispensable in diagnosis and treatment of coronary artery disease. For a number of decades, quantitative coronary analysis from selective contrast angiography (QCA) represented the state of the art in clinical applications. Several computer-based systems for quantification of local lesions have been developed during this time and are still widespread in clinical use [1]-[8]. However, it became apparent that systems based on a single projection could not provide reliable data in the common cases of foreshortening or overlapping.

Consequently, there was an extension from the projectionbased 2-D measurements into the 3-D space. Spatial reconstructions from biplane angiograms evolved as important tools for morphological analyses of vessel trees in both coronary and cerebral domains [9]-[19]. From the known imaging geometry and based on the epipolar constraint, any point visible in both projections can be spatially reconstructed by retracing the projection rays back to the point of their intersection. Since the reconstructed rays often miss this point due to slight reconstruction or calibration errors, usually their closest location is estimated as approximation (Fig. 1). High-level systems allow

This work has been supported in part by grants Pr 507/1-2 and Wa 1280/1-1 of the Deutsche Forschungsgemeinschaft [German Research Society], Bonn, Germany, and by grants IA-94-GS-65 and IA-96-GS-42 of the American Heart Association, Iowa Affiliate. Asterisk indicates corresponding author.

${ }^{*}$ A. Wahle is with the University of Iowa, Department of Electrical and Computer Engineering, Iowa City, IA 52242, USA (e-mail: a.wahle @ieee.org).

G.P.M. Prause was with the University of Iowa, Department of Electrical and Computer Engineering, Iowa City, IA 52242, USA. He is now with the MeVis Institute at the University of Bremen, D-28359 Bremen, Germany.

S. C. DeJong is with the University of Iowa, Department of Internal Medicine, Iowa City, IA 52242, USA.

M. Sonka is with the University of Iowa, Department of Electrical and Computer Engineering, Iowa City, IA 52242, USA. accurate volumetric measurements and an indirect assessment of diffuse alterations from the morphological relationships between the vessels within the arterial tree [20]-[23]. A major drawback of many of these systems is their assumption of elliptical cross-sections. Binary reconstruction methods, which allow the modeling of free-shaped contours from densitometric profiles, are well-established in ventricle analyses [24]. However, due to the limited resolution of angiography they are rarely used to assess smaller vessels like coronary or cerebral arteries [25]-[27]. As proposed in [28], the geometrically derived elliptical shape may serve as a basis for binary reconstruction methods to refine the cross-sectional contour.

Another modality, intravascular ultrasound (IVUS), was introduced carrying the promise to overcome the shortcomings of angiography. By inserting into the vessel a catheter with an ultrasonic transducer in its tip, the exact cross-sectional shape of the lumen can be visualized and quantified, and the thickness and composition of the vessel wall and plaque can be determined [29]-[36]. Acquisitions in 3-D are possible by pulling the catheter tip back during imaging, thus generating a series of images at different locations. A new problem arose: IVUS itself does not provide any information about the location of a specific image or about its spatial orientation. Common systems simply perform a straight stacking of adjacent frames, completely neglecting the influence of the vessel curvature (Fig. 2, see also [37], [38]).

This paper describes a comprehensive system for fusion of both modalities, i.e. combining the geometrical information as obtained from biplane angiography with the volumetric data derived from intravascular ultrasound. The catheter path is extracted and reconstructed from the biplane angiograms and used to map the IVUS images to their locations (Figs. 3,4). Aside from the localization of the individual IVUS frames in 3-D, the estimation of their spatial orientations is of major importance. Examples of previous work performed in this area include Laban et al. [39], Evans et al. [40], Pellot et al. [41], and Shekhar et al. [42].

Our approach incorporates several well-established algorithms for vessel detection in angiograms, for geometrical 3-D reconstruction, and for IVUS segmentation. It includes a novel method for the estimation of the frame orientation, which first determines the relative relations between adjacent IVUS frames and then optimizes the absolute orientation of the entire frame set. The methods have been validated in computer and phantom models, as well as in in-vitro studies.

Figure 5 shows the processing steps for the angiographic images (described in Section II) and the IVUS data (Section III), 
which are performed in parallel. Their output is then used for the actual fusion process (Section IV).

\section{BIPLANE ANGIOGRAPHY}

\section{A. Acquisition and Preprocessing}

The first step in angiographic processing consists of the acquisition and digitization of the images. Despite the fact that many angiographic devices provide digital output in standardized formats, the $35 \mathrm{~mm}$ cine film is still a major archiving medium. This implies that the images may have to be digitized after selection.

It is well-known from conventional QCA that the imaging process introduces numerous geometrical distortions on angiograms which need to be rectified [6], [43]. In addition, the elimination of distorting axial rotations and shifts is required for 3-D reconstruction purposes [44],[45]. For more sophisticated diagnostics, e.g. volumetric measurements on the vessel lumen, the highest accuracy in image rectification is required. For the fusion approach, we only need a 3-D description of the catheter path, while the cross-sectional data are obtained from IVUS. Since calibration procedures are mostly unacceptable in clinical routine, we are using an 8-point dewarping method that provides sufficient accuracy without the need of separately imaging a rectification grid [46]. The eight lead markers can simply be mounted on the image intensifiers and are visible in all angiograms (Fig. 6).

\section{B. Geometry Estimation}

For the reconstruction process, it is necessary to have an accurate description of the imaging geometry. Figure 7 shows the five degrees of freedom that may be chosen on a common Type-II [9] device. The angulation is defined as a combination of two rotations. The gantry shifts result in a vector $\vec{I}$ perpendicular to the projection axis; their point of intersection defines the distances $D_{S}$ to the $\mathrm{X}$-ray source and $D_{I}$ to the corresponding image intensifier.

Our approach has been adapted from the reconstruction system previously developed at the German Heart Institute of Berlin, which is described in detail elsewhere [22], [23]. An initial geometry is obtained from the parameters as read from the device, i.e. the angulations and the total distances $D_{S}+D_{I}$. So far, the geometrical model corresponds to the definition of Wollschläger et al. [9]. Orthogonal projections are desirable, due to a higher amount of spatial information as compared to low inclination angles, but are not required. The initial geometry is refined afterwards from a set of given reference points (at least two). The reconstruction errors are analyzed for specific patterns, thus correction coefficients can be calculated and applied iteratively.

For absolute length measurements, a known reference is needed. In our phantom studies, we use a wooden ball of known size $(83.5 \mathrm{~mm}$ in diameter) with two nails as markers. The outer tips of the nails provide a known distance in 3-D space and can thus be used for absolute calibration of the scene. They serve as a reference distance for refinement of the $D_{S}: D_{I}$ ratios, which are related to the respective magnifications, and the determination of the shift vectors $\vec{I}$ (Figs. 6,7). Of course, any other markers of reasonable distance can be used for this purpose as well, e.g. markers on the imaging catheter for in-vivo studies.

\section{Extraction of the Catheter Path}

After the geometry is known, the transducer in its most distal location is interactively marked in biplane angiograms acquired before the pullback is started, as well as in a location at or proximal to the end of the pullback. To find the correct correspondences, the user is supported by inserting the projection rays as auxiliary lines into the images. Arbitrary intermediate points following the catheter between its endpoints are set for further guidance. The catheter is usually visible as a local maximum along the vessel profiles (Fig. 3) and can thus be extracted using common approaches of dynamic programming or heuristic graph search [47], [48].

Our algorithm is based on dynamic programming and allows free manipulation of the regions of interest (ROIs), which are derived from Catmull-Rom splines [49] through the guide points. In the same process, the two edges of the vessel lumen outline are extracted as well, which serve as reference for establishment of the absolute frame orientation as presented in Section IV-G.

\section{3-D Reconstruction of Trajectory}

The initial reconstruction algorithm as described in [46] has been replaced by the well-established and validated 3-D reconstruction algorithm for which details are given in [22], [23]. In contrast to the reconstruction of a single point, the reconstruction of two ray bundles resulting from the projected catheter path requires a correspondence assignment of ray pairs between the projections.

We are using a cost matrix approach, which is an enhanced version of the algorithm presented by Parker et al. [50]. As shown earlier, our algorithm works well even in difficult orientations of the vessel [23], [51]. However, while the initial algorithm required a number of corresponding guide points uniquely identified in both angiograms (e.g. vessel branches), the input for this modified approach consists only of the pullback start and end points. The algorithm is further optimized to handle large vessel segments.

\section{INTRAVASCULAR ULTRASOUND}

\section{A. Acquisition and Preprocessing}

Currently, there are two major kinds of IVUS devices available [35], mechanically driven catheters and solid-state devices. Mechanically driven catheters consist of a flexible sheath, a core which contains the transducer in its tip, and an external motor which rotates the core. Solid-state devices generate images from a transducer array and contain no moving parts. The sheathed design of mechanically driven catheters has the major advantage of a stable pullback path, since only the core is moving in the direction of the pullback and the sheath remains in its position. There are common artifacts associated with the mechanical devices, mostly caused by bending of the catheter or other types of friction [37], [52], [53]. One way to avoid these distortions is to place the motor close to the tip, thus reducing influences of the path between the imaging machine and the transducer [31]. Solid-state transducers can avoid distortions from friction effects almost completely. To ensure a constant-speed pullback, automated devices are recommended. For in-vivo studies, ECGgating and/or respiratory control is required as well [35], [54]. 


\section{B. Segmentation}

A detailed overview of the common 2-D and 3-D segmentation and reconstruction systems was recently presented in [35] Some 'classic' approaches include the segmentation method of Herrington et al. [29] based on simulated annealing, and the 3-D segmentation of Li et al. [30], which performs longitudinal as well as cross-sectional semi-automatic contour detection based on a minimum cost algorithm. A validation of volumetric quantifications on straight vessels and a feasibility study of the latter algorithm in clinical applications were performed by von Birgelen et al. [33], [34].

We are using the well-established segmentation algorithm previously described in [32], [36], which is based on a graph-search approach within a given elliptical region of interest (ROI). This ROI is automatically adapted from frame to frame. Within each image, the contours indicating the inner and outer wall borders are detected, as well as the lumen/plaque interface if applicable (Fig. 8). In addition, the plaque composition may be determined for extended analysis. The entire process is automated, except for the specification of the ROI in the first image, but may be interrupted and manually corrected at any stage. The segmented contour lists are kept in 2-D polar coordinates $(v, \phi)$.

\section{FUSION OF ANGIOGRAPHY AND IVUS}

\section{A. Outline of the Fusion Process}

It is a well-known problem that the conventional straight stacking of the IVUS pixel and segmentation data does not deliver correct results [37], [38]. Figure 2 shows the typical effects of the stacking: Due to neglecting the vessel curvature, portions of the vessel volume may be either over- or underestimated, depending on which side of the vessel they are located; since the vessel torsion is not considered, the axial rotation (twist) of the catheter leads to a wrong relation of radial segments between different images. As shown in Section IV-C, curvature and torsion of an idealized catheter can be expressed using the Frenet-Serret formulas, and are a function of the pullback path of the transducer.

The vessel course is determined from the biplane angiograms, thus for each IVUS frame, its location can be determined. It must be kept in mind that the course of the catheter is not identical with the course of the vessel [55], but the correct assignment of the IVUS data in 3-D yields correct results even if the frames are oblique to the vessel. Since a constant angiographic supervision of the pullback as proposed in [40]-[42] is not applicable in clinical cases, the location of a specific frame is directly determined from its time-stamp and the pullback speed.

The estimation of the absolute orientation of the IVUS frames in 3-D is a challenging problem. While Evans et al. [40] neglected axial catheter rotations completely, the iterative methods of Pellot et al. [41] and Shekhar et al. [42] both used a local match of each individual IVUS frame with the angiographic outline. Pellot, however, refined the preliminary contour by using the densitometric profiles and a-priori information as input for a combination of Markov random fields and adapted simulated annealing [28], [41]. In contrast to determining the orientation individually for each frame, constraints from the Frenet-Serret formulas can be used to determine the twist between the frames.
Laban et al. [39] implemented the Frenet-Serret formulas directly, using a Fourier function to approximate the catheter path. This function satisfied the requirement of a third-order derivative as implied by the rules. However, the Frenet-Serret formulas can only deliver the relations between adjacent images relative to each other. What remains to be determined is the absolute orientation of the set of frames, which Laban performed iteratively by backprojection of the segmented contours and visual matching of the IVUS data in the angiograms.

We have developed a non-iterative approach for the determination of the absolute orientation, which combines the analytical calculation of the catheter twist based upon the Frenet-Serret formulas with a global optimization of the absolute frame orientation over the entire frame set.

\section{B. Localization of IVUS Frames on 3-D Path}

Our approach uses the length determination as described in [22], [23] for assigning the IVUS frames along the catheter path. In contrast to a vessel, which is of variable diameter and stiffness, the catheter has constant physical properties, which simplify the length measurement. An arbitrary downsampling rate can be specified, resulting in the assignment of a fixed number of images per millimeter pullback. In the current version, a constant pullback speed is assumed, since landmarks for synchronization are frequently not available or may be unreliable [46]. If such landmarks (e.g. clips) are available, the speed between adjacent synchronization points is assumed to be constant. For the invivo case, ECG-gated image acquisition in combination with a constant pullback have to be synchronized with the heart phase, which is subject to certain variabilities. Thus, the acquired frames would no longer be equidistant. To satisfy this condition, the algorithm is easily extendible to consider non-uniform distances between adjacent frames.

\section{Catheter Curvature and Torsion}

According to a fundamental theorem of differential geometry, a space curve with non-zero curvature is determined up to an Euclidean transformation by its curvature and torsion [56], [57].

Let $c:[0,1] \rightarrow \mathcal{R}^{3}, c(s)=[x(s), y(s), z(s)]$ be the pullback path of the transducer as a function of the arc length $s$. The local behavior of the space curve $c$ may be described by the Frenet frame, a righthanded trihedron of three orthonormal vectors $\vec{t}$ (tangent), $\vec{n}$ (normal), and $\vec{b}$ (binormal). The Frenet-Serret formulas express the local change of the Frenet frame in terms of the frame itself:

$$
\begin{aligned}
\vec{t}^{\prime}(s) & =+\kappa(s) \vec{n}(s) \\
\vec{n}^{\prime}(s) & =-\kappa(s) \vec{t}(s)+\tau(s) \vec{b}(s) \\
\vec{b}^{\prime}(s) & =-\tau(s) \vec{n}(s)
\end{aligned}
$$

where the prime denotes the derivative with respect to arc length $s$. The curvature $\kappa$ and the torsion $\tau$ are the angular velocities of the vectors $\vec{t}$ and $\vec{b}$, respectively, as the Frenet frame is moved along $c$ according to $s$. They calculate to:

$$
\begin{aligned}
\kappa(s) & =\left\|c^{\prime \prime}(s)\right\| \\
\tau(s) & =\operatorname{det}\left[c^{\prime}(s), c^{\prime \prime}(s), c^{\prime \prime \prime}(s)\right] / \kappa^{2}(s)
\end{aligned}
$$


Based upon this theory, we have developed an analytical catheter model as well as a sequential triangulation method for determination of catheter curvature and torsion during the pullback.

\section{Analytical Model of the Catheter}

The behavior of an imaging catheter was estimated analytically, resulting in the following geometrical model: We assume that the catheter consists of a chain of generalized joints, which are torsion-free (i.e. do not introduce any other axial rotation on bending than specified by the Frenet-Serret formulas). Each joint performs a relative transformation, resulting in an absolute position and orientation after applying all transformations on an initial position of the transducer. Furthermore, some constraints are given. The imaged frame is always perpendicular to the catheter axis, and the initial axial orientation is fixed (i.e. no axial twist is applied). Thus, three parameters are given for each of these joints. The length $t$ describes the distance of the endpoints when the joint is straight, an angle $\vartheta$ gives the amount of bending at this joint, and a second angle $\omega$ specifies the axis of rotation. A non-discrete version of this model can be derived for $t \rightarrow 0$, yielding an infinite number of joints within the chain.

As shown in Fig. 9, the catheter segment between two adjacent points $C_{i-1}$ and $C_{i}$ can be described by the following transformations, considering that each point is associated with both location and orientation:

1. the previous location is translated by $t_{i} / 2$;

2. the orientation is axially rotated by $+\omega_{i}$;

3 . the orientation is rotated by $\vartheta_{i}$ around a fixed axis local to the current orientation (i.e. the rotation axis was affected by the previous axial rotation);

4. the axial rotation is compensated by applying $-\omega_{i}$;

5 . the location is translated by another $t_{i} / 2$.

Equations (6)-(8) show the resulting transformation matrices using homogeneous coordinates.

$$
\begin{aligned}
C_{0} & =[x, y, z, h]=\left[\frac{x}{h}, \frac{y}{h}, \frac{z}{h}\right] \\
C_{i} & =C_{i-1} \cdot \mathbf{M}\left(\vartheta_{i}, \omega_{i}, t_{i}\right) \\
& =C_{0} \cdot \prod_{j=1}^{i} \mathbf{M}\left(\vartheta_{j}, \omega_{j}, t_{j}\right)
\end{aligned}
$$

The local curvature $\kappa_{i}$ in joint $i$ is directly related to $\vartheta_{i}$, while the local torsion $\tau_{i}$ depends on the difference $\Delta \omega_{i}$ between bending directions $\omega_{i}$ and $\omega_{i-1}$ of adjacent joints.

\section{E. Estimation of the Relative Twist}

In accordance with the catheter model described in the previous section, a sequential triangulation method is used to determine the relative twist between adjacent IVUS frames (Fig. 10). This can be considered as a discrete approximation of the FrenetSerret formulas where the local torsion $\tau_{i}$ is calculated as the angle between the normal vectors of two adjacent triangles in the reconstructed pullback polygon.

Each frame is defined by the location of its center and a local 2-D coordinate system. For time instances $i$ and $i+1$, both frames are located halfway between three consecutive points
$P_{i}, P_{i+1}, P_{i+2}$ so that:

$$
\begin{aligned}
S_{i} & =\left(P_{i}+P_{i+1}\right) / 2 \\
S_{i+1} & =\left(P_{i+1}+P_{i+2}\right) / 2
\end{aligned}
$$

The frames are perpendicular to the tangent vectors:

$$
\begin{aligned}
\vec{t}_{i} & =P_{i+1}-P_{i} \\
\vec{t}_{i+1} & =P_{i+2}-P_{i+1}
\end{aligned}
$$

The center of the circumscribed circle of the triangle as defined by:

$$
T_{i}=\left(P_{i}, P_{i+1}, P_{i+2}\right)
$$

is determined as the intersection of the perpendicular bisectors of the tangent vectors $\vec{t}_{i}$ and $\vec{t}_{i+1}$. The orientation of frame $i+1$ is determined by rotating frame $i$ by the enclosed angle $\alpha_{i}$ (which reflects the local curvature $\kappa_{i}$ ) around the normal vector:

$$
\vec{n}_{i}=\vec{t}_{i} \times \vec{t}_{i+1}
$$

of the triangle $T_{i}$. Finally, the center of frame $i+1$ is shifted to point $S_{i+1}$. In the special case of the collinearity of $\left(P_{i}, P_{i+1}, P_{i+2}\right)$, the circle has an infinite radius, i.e. $\kappa_{i}=0$, and thus no twisting takes place.

\section{F. Quantification of the Relative Twist}

Following the calculation of the catheter twist and the changes in IVUS frame orientation, all frames have a fixed orientation relative to the first frame in terms of a scalar twisting angle, even though the absolute orientation is yet unknown. To estimate the amount of twisting, i.e. the presumed error if the torsion is not considered during the reconstruction, we are using a reference plane (Fig. 11).

This reference plane is generated by bilinear regression from the catheter path data and is thus the plane of best approximation in a least square sense. The torsion-loaded path is then projected onto this plane, creating another path that retains the curvature of the catheter, but which is torsion-free. The frames for the torsion-free copy are generated in the same way as performed for its torsion-loaded original. Afterwards, the 2-D orientation vectors of the torsion-loaded path are projected onto the corresponding torsion-free frame, and their difference angle determined. Finally, the difference angles are normalized to be zero for the first frame of the pullback.

\section{G. Estimation of the Absolute Orientation}

After establishing the relative orientation changes between the frames, the absolute orientation in 3-D remains ambiguous. This problem is comparable to fitting a sock on a leg [39]: While the leg (catheter path) is stable, the sock (axial orientation of the frame set) can be freely rotated around the leg, but fits optimally only in one orientation. Our method uses the bending behavior of the imaging catheter as a reference, which is expected to fall in the position of minimum energy within the vessel [55]. This results in an out-of-center position of the catheter relative to the inner lumen, which is visible in both angiographic and IVUS data (Figs. 3,8). Based on this out-of-center position, a correction angle can be calculated. 


$$
\mathbf{M}(\vartheta, \omega, t)=\left[\begin{array}{cccc}
\cos \vartheta & -\sin \vartheta \sin \omega & -\sin \vartheta \cos \omega & 0 \\
\sin \vartheta \sin \omega & \cos \vartheta \sin ^{2} \omega+\cos ^{2} \omega & (\cos \vartheta-1) \sin \omega \cos \omega & 0 \\
\sin \vartheta \cos \omega & (\cos \vartheta-1) \sin \omega \cos \omega & \cos \vartheta \cos ^{2} \omega+\sin ^{2} \omega & 0 \\
\frac{t}{2}(\cos \vartheta+1) & -\frac{t}{2} \sin \vartheta \sin \omega & -\frac{t}{2} \sin \vartheta \cos \omega & 1
\end{array}\right]
$$

First, the segmented IVUS lumen contour is mapped into 3-D using an initial orientation. For each frame location, the angiographic outline is reconstructed as an elliptical contour as described in [22], [23], and mapped onto the IVUS frame at the respective transducer location (Fig. 12). For both angiographic and IVUS outlines, 3-D out-of-center vectors are generated from the contour center to the catheter position:

$$
\begin{aligned}
\overrightarrow{\Delta a} & =p_{\text {cath }}-p_{\text {center }} \\
\overrightarrow{\Delta v} & =m_{\text {cath }}-m_{\text {centroid }}
\end{aligned}
$$

where $\overrightarrow{\Delta a}$ is derived from the angiographic reconstruction of the catheter point $p_{\text {cath }}$ and the ellipse center $p_{\text {center }}$, and $\overrightarrow{\Delta v}$ is derived from the mapped IVUS frame center $m_{\text {cath }}$ indicating the catheter location and the centroid $m_{\text {centroid }}$ of the contour. For each frame $i$, the out-of-center strength $\mu_{i}$ and a signed error angle $\varphi_{i}$ with respect to the initial orientation can be calculated:

$$
\begin{aligned}
\mu_{i} & =\left\|\overrightarrow{\Delta v_{i}}\right\| \\
\varphi_{i} & =\angle\left(\overrightarrow{\Delta a_{i}}, \overrightarrow{\Delta v_{i}}\right)
\end{aligned}
$$

Within a moving window of arbitrary but fixed width $w$, a statistical analysis is performed. For $n_{f}$ frames, $n_{w}=n_{f}-(w-1)$ window locations exist. For each window location $k$, the summed out-of-center strength $\Sigma \mu_{k}$, the weighted mean $\bar{\varphi}_{k}$, and the weighted standard deviation $\sigma\left(\varphi_{k}\right)$ of the difference angle are calculated:

$$
\begin{aligned}
\Sigma \mu_{k} & =\sum_{i=k}^{k+(w-1)} \mu_{i} \\
\bar{\varphi}_{k} & =\frac{1}{\Sigma \mu_{k}} \sum_{i=k}^{k+(w-1)} \mu_{i} \varphi_{i} \\
\sigma\left(\varphi_{k}\right)^{2} & =\frac{1}{\Sigma \mu_{k}} \sum_{i=k}^{k+(w-1)} \mu_{i}\left(\varphi_{i}-\bar{\varphi}_{k}\right)^{2}
\end{aligned}
$$

From these values, a reliability weight is calculated for each location of the moving window, giving higher weight to locations with high out-of-center strength (i.e. locations with increased significance for the estimation of the correction angle), and limiting those with a high standard deviation of the difference angle function (i.e. locations showing distortions in either angiographic or IVUS lumen outlines):

$$
r_{k}=\frac{\Sigma \mu_{k}}{\sigma\left(\varphi_{k}\right)}
$$

A single correction angle $\bar{\varphi}_{\text {corr }}$ is determined and applied to all IVUS frames relative to the initial orientation:

$$
\Sigma r=\sum_{k=0}^{n_{w}-1} r_{k}
$$

$$
\bar{\varphi}_{\text {corr }}=\frac{1}{\Sigma r} \sum_{k=0}^{n_{w}-1} r_{k} \bar{\varphi}_{k}
$$

\section{H. 3-D Mapping of the IVUS Data}

The frames generated during the previous steps may be used in many ways: For a fast visualization, which does not even require a segmentation of the IVUS data, the pixel data are mapped into the frames; furthermore, the segmented contour and plaque composition information can be mapped into 3-D space as well by locating their corresponding pixels. The points of the segmented and mapped IVUS contours can be connected to a surface model by triangulation and then be displayed, e.g. by using the standardized Virtual Reality Modeling Language (VRML) with a respective viewer.

A voxel cube of pre-defined size can be generated and used as input for common volume-oriented systems. Therefore, the 3-D space is divided into cubic voxels. After assigning a specific IVUS pixel to its 2-D coordinates, and mapping into 3-D space, the corresponding voxel can be determined. Depending on the size of the cube, it may occur that two or more IVUS pixels share the same voxel, or that a single IVUS pixel extends over more than one voxel.

The resolution of the voxel cube depends on several factors. The basic limitation is the resolution of the image data (both angiographic and IVUS), as well as the distance between the frames. Furthermore, the dimensions of the voxel cube are restricted by computer performance and memory. Typical sizes are $380 \times 380 \times 380$ voxels, e.g. allowing a resolution around 150 $180 \mu \mathrm{m}$ for a curved vessel segment of $100-120 \mathrm{~mm}$ length, or of $50 \mu \mathrm{m}$ for assessing a local lesion of $12-15 \mathrm{~mm}$ length.

\section{VALIDATION}

\section{A. Computer Simulations}

For validation in an idealized environment, computer simulations were performed. Since the algorithm was tested on regular structures like helices before [55], we focused on models of known, but irregular bending. The first model consisted of five generalized joints as described in Section IV-D. To check its correctness, a physical model with five joints was built and supplied with markers indicating the orientation of the local 2-D coordinate systems. Both computer and physical models were bent with the same sets of angles $\left(\omega_{i}, \vartheta_{i}\right)$. Figure 13a shows some examples. In the following step, the sequential triangulation algorithm was applied to the 3-D path as indicated by points $P_{i}$, and the matched 2-D coordinate systems $\left(U_{i}, V_{i}\right)$ were compared with the originally computed ones.

To simulate the non-discrete case, an irregular path was generated. Two sine-functions of different amplitudes and frequencies were applied to the angles $\omega$ and $\vartheta$, thus changing both local curvature and torsion in every joint. A total path of $10 \mathrm{~cm}$ length was 
generated, and a joint was placed at each $100 \mu \mathrm{m}$. Ten equidistant reference coordinate systems were calculated. The 3-D path was downsampled by a factor of 5 to achieve the angiographic resolution of $500 \mu \mathrm{m}$ and to blur the path (Fig. 13b). Finally, the catheter twist was determined with our sequential triangulation algorithm and the resulting 2-D coordinate systems compared with the previously modeled ones by calculating the difference angles between their unit vectors.

\section{B. Data Acquisition}

For the phantom and in-vitro studies, we utilized a biplane Philips device for angiography, with a diagonal of 9" at image intensifier level. The $35 \mathrm{~mm}$ cine films were digitized on a Nikon Coolscan, ${ }^{\mathrm{TM}}$ which was set to a square window with a size of $512 \times 512$ pixels. The resulting resolution was 0.33 $0.35 \mathrm{~mm} / \mathrm{pixel}$ in image intensifer level, which corresponds to a resolution of $0.20-0.22 \mathrm{~mm} / \mathrm{pixel}$ in object level.

IVUS imaging was performed using standard mechanically driven catheters (MicroView, ${ }^{\mathrm{TM}} 2.9 \mathrm{~F}, 30 \mathrm{MHz}$, of CVIS, Sunnyvale CA; and UltraCross, ${ }^{\mathrm{TM}} 3.2 \mathrm{~F}, 30 \mathrm{MHz}$, of SCIMED, Maple Grove MN; with either CVIS Insight ${ }^{\mathrm{TM}}$ or HP Sonos ${ }^{\mathrm{TM}}$ consoles). The pullback was performed either by an automatic pullback device (CVIS), or manually by an experienced operator (S.C. DeJong), with a desired speed of $1.0 \mathrm{~mm} / \mathrm{s}$. Images were recorded on S-VHS tapes and digitized afterwards in $640 \times 480$ size. For each image, a $380 \times 380$ subimage was extracted, containing the relevant data with a resolution of $40-55 \mu \mathrm{m} / \mathrm{pixel}$ and the transducer location in the image center.

\section{Torsion-Free Environment}

An important test is the verification of the assumption of a torsion-free catheter. If this assumption is correct, the orientation of the IVUS image should remain constant if the catheter is moved and bent within a stable plane. For this purpose, we supplied a wooden plate with guide nails (Fig. 14). Because the wood does not deliver a sufficient ultrasonic echo, its top surface was covered with an aluminum foil. Furthermore, the plate was submerged in a water bath, and the path of the catheter between the motor unit and the beginning of the plate was fixed during imaging. In this specific experiment, the pullback was performed with the automatic pullback device to eliminate any influence from the manual handling of the catheter. Three setups were imaged: (a) the catheter was straight along the plate, (b) the catheter was slightly bent over the upper restraint point, and (c) the catheter was bent in a double- $S$ shape using the three lower nails. In each pullback, the axial rotations of the images were analyzed.

\section{Cadaveric Pig Hearts}

The right coronary arteries of two fresh cadaveric pig hearts were supplied with eight straight clips each, allowing a proper identification in both angiographic and IVUS images (Figs. 15,16). The vessels were catheterized and pressurized with $0.9 \%$ saline at about $100 \mathrm{mmHg}$. Each pig heart was immersed into a cylindrical container $(100 \times 200 \mathrm{~mm})$, which was filled with water at body temperature. The IVUS catheter was inserted into the coronary artery and advanced into position under fluoroscopic control with help of a guidewire, which was removed afterwards. Diluted contrast agent was injected into the coronary artery and biplane angiograms of the pig heart were recorded for 3-5 seconds on cine film, showing the transducer in its distal location. The IVUS catheter was pulled back manually at approximately constant speed of $1.0 \mathrm{~mm} / \mathrm{s}$. Three pullbacks per heart were recorded over a length of $110-130 \mathrm{~mm}$, resulting in approximately 3400 images per pullback. In one of the hearts, the most proximal clip could not be reached in two of three pullbacks and this marker was therefore discarded from the analysis.

From the angiograms, both the catheter path and the catheter clips were reconstructed and retained in separate 3-D models. The clips were also used for the imaging geometry refinement. For each clip, its closest distance to the catheter was determined in order to generate a 3-D vector indicating the expected orientation of the IVUS peak. Between adjacent clips, the actual pullback speed was estimated from the reconstruction and the IVUS time-stamps, and the location errors were corrected. The peaks were mapped into 3-D space in accordance with the calculated twist, and the angular errors between the mapped peaks and those previously reconstructed from the angiograms were calculated. The absolute orientation was corrected to minimize the mean angular error over all peaks within a pullback.

In a third cadaveric pig heart without clips, the complete fusion was performed including IVUS segmentation (Figs. 2b, 3, 8). The absolute orientation was determined both interactively and by our algorithm. Afterwards, the resulting vessel surfaces as well as the catheter path were reprojected into the angiograms and visually compared with the lumen profiles.

\section{RESULTS}

\section{A. Computer Simulations}

A visual comparison of the computed discrete 5-joint model with the physical one showed a good match of the $\left(U_{i}, V_{i}\right)$ vectors and thus qualitatively confirmed the correctness of the transformation chain. The root mean square (RMS) errors in axial orientation between the modeled frames and those predicted from the pure path was $0.004^{\circ}$ over all selected positions, with a maximum error of $0.010^{\circ}$. In the downsampled quasi nondiscrete model of the two sine waves, an RMS error of $1.054^{\circ}$ along with a maximum error of $2.521^{\circ}$ could be measured from the ten references.

\section{B. Torsion-Free Environment}

In the phantom study using the nail plate, the catheter showed no significant twisting $\left(<3^{\circ}\right)$ during pullback in the straight setup. On slight curvature with weak restraint (approx. $32^{\circ}$ around the upper nail), no effects caused by bending were detected. The axial orientation between images in the center of the segments proximal and distal to the nail differed by less than $1^{\circ}$. Thus, the catheter behaved as expected.

However, when using the three lower nails and a stronger restraint, significant twisting was observed. Figure 17 shows the echoes at the most proximal nail, where the catheter is bent by approx. $50^{\circ}$ along a segment of $15 \mathrm{~mm}$. Theoretically, the orientation of the echo should remain constant at all locations 
despite of the bending. In this extreme example, the echo covered a radial segment of $57^{\circ}$. The difference of the orientation between the distal and the proximal end-points of the curve was $9^{\circ}$, where the proximal echo moved counter-clockwise compared to the distal one. In addition to the clockwise rotation, non-uniform rotational distortions (NURDs) could be identified, indicating varying rotational velocity of the catheter core. The enclosed angle of the foil echoes left and right to the catheter should always be $180^{\circ}$. However, at the closest distance to the nail, the enclosed angle was as low as $115^{\circ}$. The mentioned effects could be measured at the other two nails as well, although in less strength (clockwise rotations of $42^{\circ}$ at the distal and $19^{\circ}$ at the center nail). For the NURD effects, the enclosed angle at the center nail was increased, while it was decreased at the outer nails.

A further distortion could be recognized when saline was flushed into the catheter. The short increase of the pressure within the catheter introduced a clockwise axial rotation by up to $70^{\circ}$ during the flush.

\section{Clips Analysis}

In contrast to the previously described phantom study, the pullbacks were performed manually in the cadaveric pig hearts. From the 40 segments between adjacent clips over all three pullbacks in each heart, the real speed was calculated as $1.14 \mathrm{~mm} / \mathrm{s}$ with a standard deviation of $0.34 \mathrm{~mm} / \mathrm{s}$, ranging $0.55-2.33 \mathrm{~mm} / \mathrm{s}$.

For 45 clips, the orientations of the angiographically reconstructed peak vectors and those mapped from IVUS were compared. The RMS error of the predicted frame orientations over all six pullbacks was $21.96^{\circ}$ with a standard deviation of $4.87^{\circ}$. In comparison, the mathematical analysis of the pullback trajectory revealed that the catheter was twisting up to $60^{\circ}$ (Fig. 18). After adjustment of the absolute orientation to minimize the overall mean matching error, and generation of the voxel cube, the visual results showed a good correspondence (Fig. 19).

We performed further evaluations on this data set to identify the possible sources for the mapping errors. We compared the reproducibility of the manual pullbacks from the relative 2-D twist between images of adjacent clips, resulting in an acceptable RMS error of $5.01^{\circ}$ over all 39 pairs. However, the maximum of $27.5^{\circ}$ showed that local tolerances might be quite high. The major reasons for the remaining errors are most likely caused by the effects described in Section VI-B.

\section{Complete Fusion Cycle}

The reconstruction from the third pig heart showed a good performance for both angiographic and IVUS parts. The comparison of the projected segmentation results with the lumen profiles visible in the angiograms showed a good match. Slight tolerances could be identified, which were obviously caused by localization errors due to the manual pullback. Since these tolerances distorted the results of the absolute orientation determination, the erroneous segments were manually discarded. This caused a change of $33.6^{\circ}$ in absolute frame orientation compared to the unmodified calculation. In result, there was no visible difference between the interactively matched and calculated frame orientation.

\section{DISCUSSION}

The methods for data fusion between biplane angiography and IVUS reported in this paper showed a good overall performance. The computer simulations demonstrated an excellent reconstruction of the spatial orientations of the IVUS frames, even in the case of an $80 \%$ loss of 3-D information by downsampling. Aside from computer simulations, quantitative validation could only be performed in-vitro. To the extent of our knowledge, there is no reliable independent standard (e.g. objective measurements or manual tracing by an experienced user) for an in-vivo validation of this type of fusion method. However, the underlying methods for the angiographic reconstruction and IVUS segmentation have been extensively validated in-vitro and in-vivo.

The evaluation of the algorithm for 3-D reconstruction from biplane angiograms confirmed its good accuracy reported earlier [22], [23]. The marking process is simplified substantially, because the interactively set intermediate guide points along the pullback path no longer require a correspondence between both projections. However, problems may still occur if the cost matrix approach is applied to self-intersecting or highly ambiguous vessels. Thus, possible future extensions may include the development of a knowledge-based graph-oriented search along epipolar planes [58], or may utilize active contours (snakes) for extraction of the catheter path in the angiograms and the 3-D reconstruction in a single step [59].

While the automated pullback used for the phantom showed a good stability of the pullback speed, the manual pullback performed in the pig hearts was subject to several distortions. If a constant pullback speed is assumed, this leads to significant errors in the matching of the estimated locations of the frames, and thus to miscalculations of the local axial twist as well. The high variance in the pullback speed proved that the manual pullback is not acceptable for high-quality assessments such as 3-D reconstructions and volumetric quantifications. On the other hand, these artifacts are avoidable with moderate effort, e.g. by using a motorized pullback device.

Currently, the algorithm assumes a stable path of the catheter during pullback. While this is true for sheathed catheters, the tip of an unsheathed catheter will probably not follow the path as derived from the angiographic images depicting the transducer in its distal position. Thus, the approach presented here is limited to sheathed catheters. For in-vivo applications, problems with ECG and respiratory gatings occur, and non-uniform distances between adjacent frames have to be considered. These items need to be addressed by future developments.

The matching of the clip peaks showed a good estimation of the orientations of the frames, but uncovered some limitations as well. Mechanically driven imaging catheters are sensitive to bending and external forces. Friction effects may introduce a phase shift, resulting in an artificial axial rotation of the images. Changing the pressure in the catheter, e.g. by a routine saline flush, introduces similar effects. In-vivo, the slight pressure changes introduced by blood flow are unlikely to affect the catheter core inside the sheath. The detected phase shifts consist of both local and additive components. The local component causes a rotation that is present only at the restraint point itself, while the additive component affects the entire catheter core distal to the restraint point. For the catheters we used, these effects 
resulted always in a clockwise rotation of the echoes. However, the NURD effects depend on the direction of bending.

We assume that the linear over-estimation of the catheter twist as measured in helical phantoms earlier [55] was caused by the friction of the core against the sheath, which was equally distributed due to the constant curvature of the helix. The manual pullback may introduce further distortions if the catheter core is twisted against the sheath. With future extensions to the method for establishing the absolute orientation of the frames, friction points can be identified from inconsistencies in the angle function $\varphi$, thus creating multiple correction angles for the specific vessel segments.

\section{CONCLUSIONS}

We have developed and validated a comprehensive system for a geometrically correct 3-D reconstruction of coronary arteries based on fusion of IVUS and biplane angiography. Existing wellestablished systems for both IVUS segmentation and geometrical reconstruction from biplane angiograms have been incorporated. The use of an automated pullback device is inevitable in order to obtain an accurate reconstruction of the transducer's trajectory and a correct determination of the IVUS frames. Although our method showed excellent results in computer simulations, the invitro application in phantoms and cadaveric pig hearts uncovered influence from several sources of distortion, mainly caused by mechanical components of the setup. The systematic nature of these distortions holds a substantial promise for the successful development of an automated correction procedure.

\section{REFERENCES}

[1] B. G. Brown, E. L. Bolson, M. Frimer, and H. T. Dodge, "Quantitative coronary arteriography; estimation of dimensions, hemodynamic resistance, and atheroma mass of coronary artery lesions using the arteriogram and digital computation," Circulation, vol. 55, no. 2, pp. 329-337, Feb. 1977.

[2] R. L. Kirkeeide, P. Fung, R. W. Smalling, and K. L. Gould, "Automated evaluation of vessel diameter from arteriograms," in Proc. Computers in Cardiology 1982, Seattle WA, Los Alamitos CA, pp. 215-218, IEEE-CS Press, 1982.

[3] J. H. C. Reiber, C. J. Kooijman, C. J. Slager, J. J. Gerbrands, J. C. H Schuurbiers, A. den Boer, W. Wijns, P. W. Serruys, and P. G. Hugenholtz, "Coronary artery dimensions from cineangiograms - methodology and validation of a computer-assisted analysis procedure," IEEE Transactions on Medical Imaging, vol. MI-3, no. 3, pp. 131-141, Sept. 1984.

[4] B. G. Brown, P. Simpson, J. T. Dodge, E. L. Bolson, and H. T. Dodge, "Quantitative and qualitative coronary arteriography," in Quantitative Coronary Arteriography, J. H. C. Reiber and P. W. Serruys, eds., vol. 117 of Developments in Cardiovascular Medicine, Dordrecht, pp. 3-21, Kluwer, 1991.

[5] P. J. de Feyter, P. W. Serruys, M. J. Davies, P. Richardson, J. Lubsen, and M. F. Oliver, "Quantitative coronary angiography to measure progression and regression of coronary atherosclerosis; value, limitations, and implications for clinical trials," Circulation, vol. 84, no. 1, pp. 412-423, July 1991.

[6] J. Beier, H. Oswald, H. U. Sauer, and E. Fleck, "Accuracy of measurement in quantitative coronary angiography (QCA)," in Computer Assisted Radiology (CAR '91), H. U. Lemke, M. L. Rhodes, C. C. Jaffe, and R. Felix, eds., Berlin/New York, pp. 721-726, Springer, 1991.

[7] P. M. J. van der Zwet and J. H. C. Reiber, "A new approach for the quantification of complex lesion morphology: The gradient field transform basic principles and validation results," Journal of the American College of Cardiology, vol. 24, no. 1, pp. 216-224, July 1994.

[8] M. Sonka, M. D. Winniford, and S. M. Collins, "Robust simultaneous detection of coronary borders in complex images," IEEE Transactions on Medical Imaging, vol. 14, no. 1, pp. 151-161, Mar. 1995.
[9] H. Wollschläger, P. Lee, A. Zeiher, U. Solzbach, T. Bonzel, and H. Just, "Mathematical tools for spatial computations with biplane isocentric X-ray equipment," Biomedizinische Technik, vol. 31, no. 5, pp. 101-106, May 1986.

[10] D. L. Parker, D. L. Pope, K. S. White, L. R. Tarbox, and H. W. Marshall, "Three-dimensional reconstruction of vascular beds," in Information Processing in Medical Imaging, S. L. Bacharach, ed., Boston MA, pp. 414430, Nijhoff, 1986.

[11] G. Coppini, M. Demi, and G. Valli, "3-D reconstruction of vascular trees," in Digital Signal Processing, V. Cappellini and A. G. Constantinides, eds., pp. 911-915, Elsevier/North-Holland, 1987.

[12] K. Kitamura, J. M. Tobis, and J. Sklansky, "Estimating the 3-D skeletons and transverse areas of coronary arteries from biplane angiograms," IEEE Transactions on Medical Imaging, vol. 7, no. 3, pp. 173-187, Sept. 1988.

[13] M. Büchi, O. M. Hess, R. L. Kirkeeide, T. Suter, M. Muser, H. P. Osenberg, P. Niederer, M. Anliker, K. L. Gould, and H. P. Krayenbühl, "Validation of a new automatic system for biplane quantitative coronary arteriography," International Journal of Cardiac Imaging, vol. 5, pp. 93-103, 1990.

[14] F. Cheriet, J. Meunier, J. Lesperance, and M. Bertrand, "Self-calibration of a stereo system using matched points; application to biplane X-ray imaging," in Intelligent Robots and Computer Vision XIII: 3D Vision, Product Inspection, and Active Vision, D. P. Casasent, ed., vol. 2354, Bellingham WA, pp. 279-289, SPIE, 1994.

[15] A. M. M. Muijtjens, J. M. A. Roos, T. Arts, A. Hasman, and R. S. Reneman, "Simultaneous estimation of stereo correspondence and camera geometry from marker tracks," in Proc. Computers in Cardiology 1995, Vienna AT, Piscataway NJ, pp. 577-580, IEEE Press, 1995.

[16] K. R. Hoffmann, C. E. Metz, and Y. Chen, "Determination of 3D imaging geometry and object configurations from two biplane views: An enhancement of the Metz-Fencil technique," Medical Physics, vol. 22, no. 8, pp. 1219-1227, Aug. 1995.

[17] S. Y. J. Chen and C. E. Metz, "Improved determination of biplane imaging geometry from two projection images and its application to threedimensional reconstruction of coronary arterial trees," Medical Physics, vol. 24, no. 5, pp. 633-654, May 1997.

[18] P. M. Hall, M. Ngan, and P. M. Andreae, "Reconstruction of vascular networks using three-dimensional models," IEEE Transactions on Medical Imaging, vol. 16, no. 6, pp. 919-929, Dec. 1997.

[19] K. D. Tönnies, L. Remonda, and D. Koster, "Combining extraction and 3-D reconstruction of vessel center lines in biplane subtraction angiography," in Medical Imaging 1998: Image Processing, K. M. Hanson, ed., vol. 3338, Bellingham WA, pp. 492-503, SPIE, 1998.

[20] N. Guggenheim, P. A. Doriot, P. A. Dorsaz, P. Descouts, and W. Rutishauser, "Spatial reconstruction of coronary arteries from angiographic images," Physics in Medicine and Biology, vol. 36, no. 1, pp. 99110, Jan. 1991.

[21] C. Seiler, R. L. Kirkeeide, and K. L. Gould, "Basic structure-function relations of the epicardial coronary vascular tree; basis of quantitative coronary arteriography for diffuse coronary artery disease," Circulation, vol. 85, no. 6, pp. 1987-2003, June 1992.

[22] A. Wahle, E. Wellnhofer, I. Mugaragu, H. U. Sauer, H. Oswald, and E. Fleck, "Assessment of diffuse coronary artery disease by quantitative analysis of coronary morphology based upon 3-D reconstruction from biplane angiograms," IEEE Transactions on Medical Imaging, vol. 14, no. 2, pp. 230-241, June 1995.

[23] A. Wahle, Präzise dreidimensionale Rekonstruktion von Gefäßsystemen aus biplanen angiographischen Projektionen und deren klinische Anwendung. No. 152 in Fortschritt-Berichte, Reihe Biotechnik (17), Düsseldorf: VDI Verlag, 1997. (in German).

[24] G. P. M. Prause and D. G. W. Onnasch, "Binary reconstruction of the heart chambers from biplane angiographic image sequences," IEEE Transactions on Medical Imaging, vol. 15, no. 4, pp. 532-546, Aug. 1996.

[25] J. H. C. Reiber, J. J. Gerbrands, G. J. Troost, C. J. Kooijman, and C. H. Slump, "3-D reconstruction of coronary arterial segments from two projections," in Digital Imaging in Cardiovascular Radiology, P. H. Heintzen and R. Brennecke, eds., Stuttgart/New York, pp. 151-163, Thieme, 1982/83.

[26] Y. Bao, "A pyramidal approach to three-dimensional reconstruction of a vascular system from two projections," in Computer Assisted Radiology (CAR '89), H. U. Lemke, M. L. Rhodes, C. C. Jaffe, and R. Felix, eds., Berlin/New York, pp. 317-321, Springer, 1989.

[27] L. van Tran, R. C. Bahn, and J. Sklansky, "Reconstructing the cross sections of coronary arteries from biplane angiograms," IEEE Transactions on Medical Imaging, vol. 11, no. 4, pp. 517-529, Dec. 1992.

[28] C. Pellot, A. Herment, M. Sigelle, P. Horain, H. Maitre, and P. Peronneau, "A 3D reconstruction of vascular structures from two X-ray angiograms using an adapted simulated annealing algorithm," IEEE Transactions on Medical Imaging, vol. 13, no. 1, pp. 48-60, Mar. 1994. 
[29] D. M. Herrington, T. Johnson, P. Santago, and W. E. Snyder, "Semi-automated boundary detection for intravascular ultrasound," in Proc. Computers in Cardiology 1992, Durham NC, Los Alamitos CA, pp. 103-106, IEEE-CS Press, 1992.

[30] W. Li, C. von Birgelen, C. Di Mario, E. Boersma, E. J. Gussenhoven, N. H J. J. van der Putten, and N. Bom, "Semi-automatic contour detection for volumetric quantification of intravascular ultrasound," in Proc. Computers in Cardiology 1994, Bethesda MD, Los Alamitos CA, pp. 277-280, IEEECS Press, 1994/95.

[31] N. Bom, W. Li, A. F. W. van der Steen, C. L. de Korte, E. J. Gussenhoven, C. von Birgelen, C. T. Lancee, and J. R. T. C. Roelandt, "Intracoronary ultrasound: Technical update 1995," in Quantitative Coronary Imaging, P. J. de Feyter, C. Di Mario, and P. W. Serruys, eds., Rotterdam, pp. 89-106, Barjesteh/Meeuwes, 1995.

[32] M. Sonka, X. Zhang, M. Siebes, M. S. Bissing, S. C. DeJong, S. M. Collins, and C. R. McKay, "Segmentation of intravascular ultrasound images: A knowledge-based approach," IEEE Transactions on Medical Imaging, vol. 14, no. 4, pp. 719-732, Dec. 1995.

[33] C. von Birgelen, C. Di Mario, W. Li, J. C. H. Schuurbiers, C. J. Slager, P. J. de Feyter, J. R. T. C. Roelandt, and P. W. Serruys, "Morphometric analysis in three-dimensional intracoronary ultrasound: An in-vitro and in-vivo study performed with a novel system for the contour detection of lumen and plaque," American Heart Journal, vol. 132, no. 3, pp. 516-527, Sept. 1996

[34] C. von Birgelen, E. A. de Vrey, G. S. Mintz, A. Nicosia, N. Bruining, W. Li, C. J. Slager, J. R. T. C. Roelandt, P. W. Serruys, and P. J. de Feyter, "ECGgated three-dimensional intravascular ultrasound: Feasibility and reproducibility of the automated analysis of coronary lumen and atherosclerotic plaque dimensions in humans," Circulation, vol. 96, no. 9, pp. 2944-2952, Nov. 1997.

[35] J. Dijkstra, A. Wahle, G. Koning, J. H. C. Reiber, and M. Sonka, "Quantitative coronary ultrasound: State of the art," in What's New in Cardiovascular Imaging?, J. H. C. Reiber and E. E. van der Wall, eds., vol. 204 of Developments in Cardiovascular Medicine, Dordrecht, pp. 79-94, Kluwer, 1998.

[36] X. Zhang, C. R. McKay, and M. Sonka, "Tissue characterization in intravascular ultrasound images," IEEE Transactions on Medical Imaging, vol. 17 , no. 6 , pp. 889-899, Dec. 1998.

[37] J. R. T. C. Roelandt, C. Di Mario, N. G. Pandian, W. Li, D. Keane, C. J. Slager, P. W. de Feyter, and P. W. Serruys, "Three-dimensional reconstruction of intracoronary ultrasound images; rationale, approaches, problems, and directions," Circulation, vol. 90, no. 2, pp. 1044-1055, Aug. 1994.

[38] E. Maurincomme and G. Finet, "What are the advantages and limitations of three-dimensional intracoronary ultrasound imaging?," in Cardiovascular Imaging, J. H. C. Reiber and E. E. van der Wall, eds., vol. 186 of De velopments in Cardiovascular Medicine, Dordrecht, pp. 243-255, Kluwer, 1996.

[39] M. Laban, J. A. Oomen, C. J. Slager, J. J. Wentzel, R. Krams, J. C. H Schuurbiers, A. den Boer, C. von Birgelen, P. W. Serruys, and P. J. de Feyter, "ANGUS: A new approach to three-dimensional reconstruction of coronary vessels by combined use of angiography and intravascular ultrasound," in Proc. Computers in Cardiology 1995, Vienna AT, Piscataway NJ, pp. 325328, IEEE Press, 1995.

[40] J. L. Evans, K. H. Ng, S. G. Wiet, M. J. Vonesh, W. B. Burns, M. G Radvany, B. J. Kane, C. J. Davidson, S. I. Roth, B. L. Kramer, S. N. Meyers, and D. D. McPherson, "Accurate three-dimensional reconstruction of intravascular ultrasound data; spatially correct three-dimensional reconstructions," Circulation, vol. 93, no. 3, pp. 567-576, Feb. 1996.

[41] C. Pellot, I. Bloch, A. Herment, and F. Sureda, "An attempt to 3-D reconstruct vessel morphology from X-ray projections and intravascular ultrasounds modeling and fusion," Computerized Medical Imaging and Graphics, vol. 20, no. 3, pp. 141-151, May/June 1996.

[42] R. Shekhar, R. M. Cothren, D. G. Vince, and J. F. Cornhill, "Fusion of intravascular ultrasound and biplane angiography for three-dimensional reconstruction of coronary arteries," in Proc. Computers in Cardiology 1996, Indianapolis IN, Piscataway NJ, pp. 5-8, IEEE Press, 1996.

[43] E. Gronenschild, "The accuracy and reproducibility of a global method to correct for geometric image distortion in the X-ray imaging chain,' Medical Physics, vol. 24, no. 12, pp. 1875-1888, Dec. 1997.

[44] K. R. Hoffmann, Y. Chen, J. Esthappan, S. Y. J. Chen, and J. D. Caroll, "Pincushion correction techniques and their effects on calculated 3-D positions and imaging geometries," in Medical Imaging 1996: Image Processing, M. H. Loew and K. M. Hanson, eds., vol. 2710, Bellingham WA, pp. 462-467, SPIE, 1996.

[45] A. Wahle, U. Krauß, H. Oswald, and E. Fleck, "Inter- and extrapolation of correction coefficients in dynamic image rectification," in Proc. Computers in Cardiology 1997, Lund SE, vol. 24, Piscataway NJ, pp. 521-524, IEEE Press, 1997.
[46] G. P. M. Prause, S. C. DeJong, C. R. McKay, and M. Sonka, "Semiautomated segmentation and 3-D reconstruction of coronary trees: Biplane angiography and intravascular ultrasound data fusion," in Medical Imaging 1996: Physiology and Function from Multidimensional Images, E. A. Hoffman, ed., vol. 2709, Bellingham WA, pp. 82-92, SPIE, 1996.

[47] D. L. Pope, D. L. Parker, D. E. Gustafson, and P. D. Clayton, "Dynamic search algorithms in left ventricular border recognition and analysis of coronary arteries," in Proc. Computers in Cardiology 1984, Salt Lake City UT, Los Alamitos CA, pp. 71-75, IEEE-CS Press, 1984.

[48] M. Sonka, V. Hlavac, and R. Boyle, Image Processing, Analysis, and Machine Vision. Pacific Grove: PWS Publishing, 2nd ed., 1998/99.

[49] E. Catmull and R. Rom, "A class of local interpolating splines," in Computer Aided Geometric Design, R. E. Barnhill and R. F. Riesenfeld, eds., New York, pp. 317-326, Academic Press, 1974.

[50] D. L. Parker, D. L. Pope, R. E. van Bree, and H. W. Marshall, "Threedimensional reconstruction of moving arterial beds from digital subtraction angiography," Computers and Biomedical Research, vol. 20, no. 2, pp. 166185, Apr. 1987.

[51] A. Wahle, H. Oswald, G. A. Schulze, J. Beier, and E. Fleck, "3-D reconstruction, modelling and viewing of coronary vessels," in Computer Assisted Radiology (CAR '91), H. U. Lemke, M. L. Rhodes, C. C. Jaffe, and R. Felix, eds., Berlin/New York, pp. 669-676, Springer, 1991.

[52] H. ten Hoff, A. Korbijn, T. H. Smith, J. F. Klinkhamer, and N. Bom, "Imaging artifacts in mechanically driven ultrasound catheters," International Journal of Cardiac Imaging, vol. 4, pp. 195-199, 1989.

[53] B. J. Kimura, V. Bhargava, W. Palinski, R. J. Russo, and A. N. DeMaria, "Distortion of intravascular ultrasound images because of nonuniform angular velocity of mechanical type transducers," American Heart Journal, vol. 132 , no. 2/1, pp. 328-336, Aug. 1996.

[54] N. Bruining, C. von Birgelen, M. T. Mallus, P. J. de Feyter, E. de Vrey, W. Li, F. Prati, P. W. Serruys, and J. R. T. C. Roelandt, "ECG-gated ICUS image acquisition combined with a semi-automated contour detection provides accurate analysis of vessel dimensions," in Proc. Computers in Cardiology 1996, Indianapolis IN, Piscataway NJ, pp. 53-56, IEEE Press, 1996.

[55] G. P. M. Prause, S. C. DeJong, C. R. McKay, and M. Sonka, "Towards a geometrically correct 3-D reconstruction of tortuous coronary arteries based on biplane angiography and intravascular ultrasound," International Journal of Cardiac Imaging, vol. 13, no. 6, pp. 451-462, Dec. 1997.

[56] B. O’Neill, Elementary Differential Geometry. New York: Academic Press, 1966.

[57] G. E. Farin, Curves and Surfaces for Computer Aided Geometric Design: A Practical Guide. Boston: Academic Press, 2nd ed., 1990.

[58] P. M. Hall, "Robust reconstruction of 3-D space-curves from images at arbitrary angles," in 8th British Machine Vision Conference (BMVC '97), A. F. Clark, ed., Essex, pp. 300-309, BMVA Press, 1997.

[59] C. Molina, G. P. M. Prause, P. Radeva, and M. Sonka, "3-D catheter path reconstruction from biplane angiograms," in Medical Imaging 1998: Image Processing, K. M. Hanson, ed., vol. 3338, Bellingham WA, pp. 504-512, SPIE, 1998. 


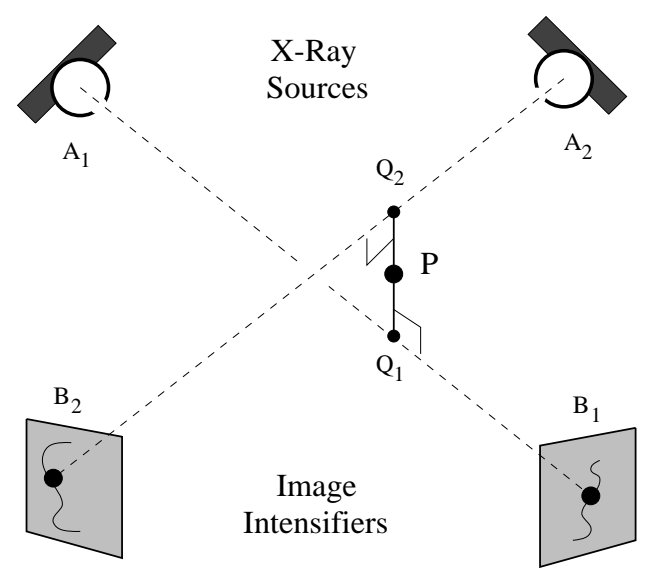

Fig. 1. Theory of 3-D reconstruction of a point from its known locations in biplane angiograms.

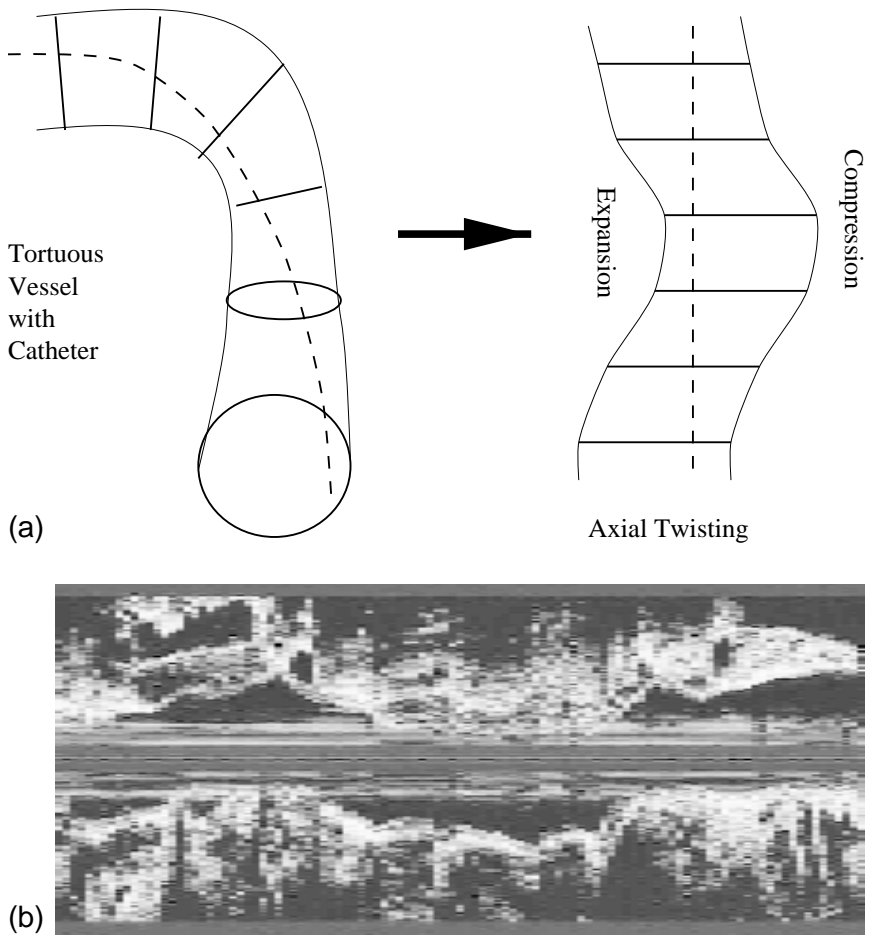

Fig. 2. Problems of the conventional reconstruction methods that use a straight stacking of the IVUS slices without consideration of the vessel curvature; (a) theory, (b) example.
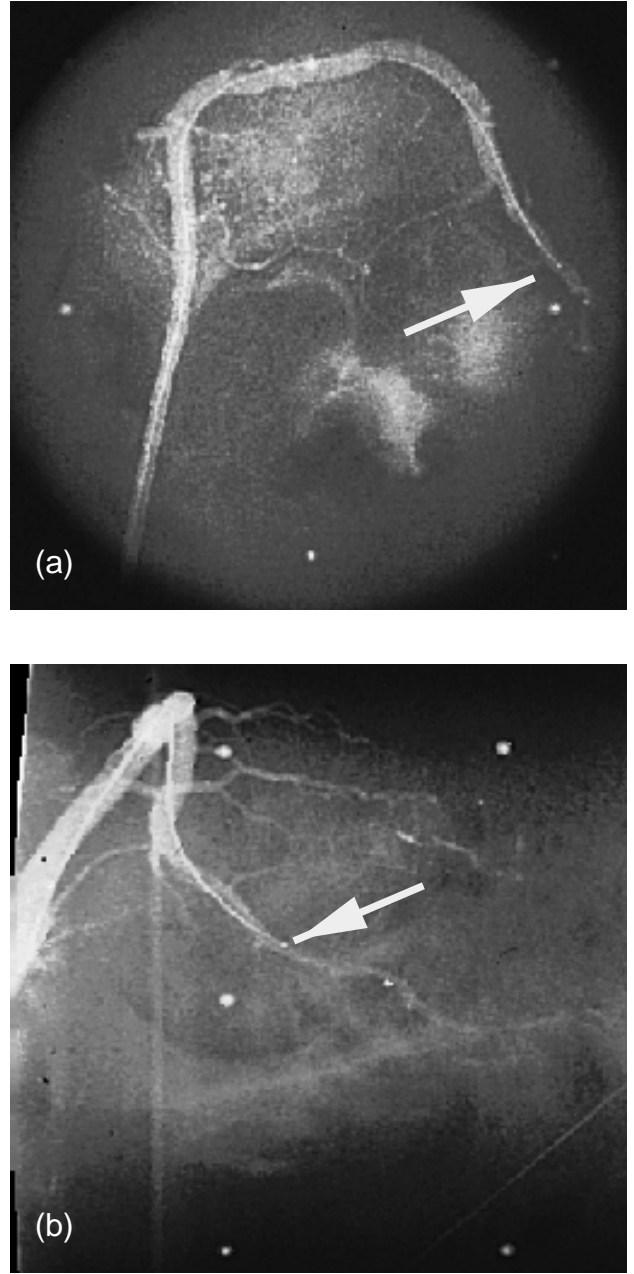

Fig. 3. Biplane angiograms of a coronary artery in a cadaveric pig heart; (a) frontal and (b) lateral projections, with inserted IVUS catheter (arrows indicate the location of the tip of the catheter core).

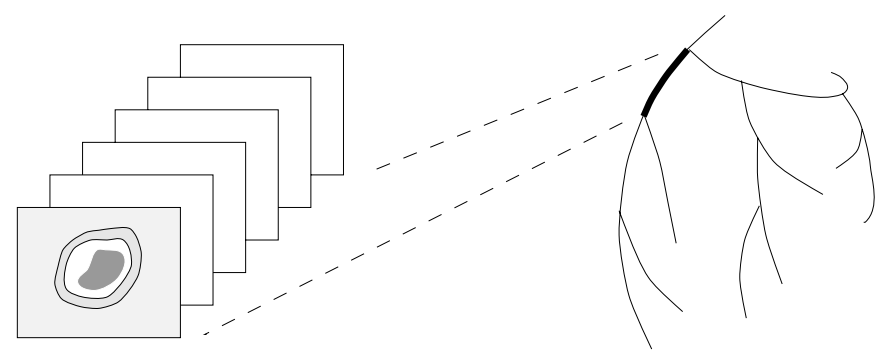

Fig. 4. Image fusion between angiography and IVUS facilitates a geometrically correct assignment of the IVUS images to the vessel segment in both 3-D locations and orientations. 


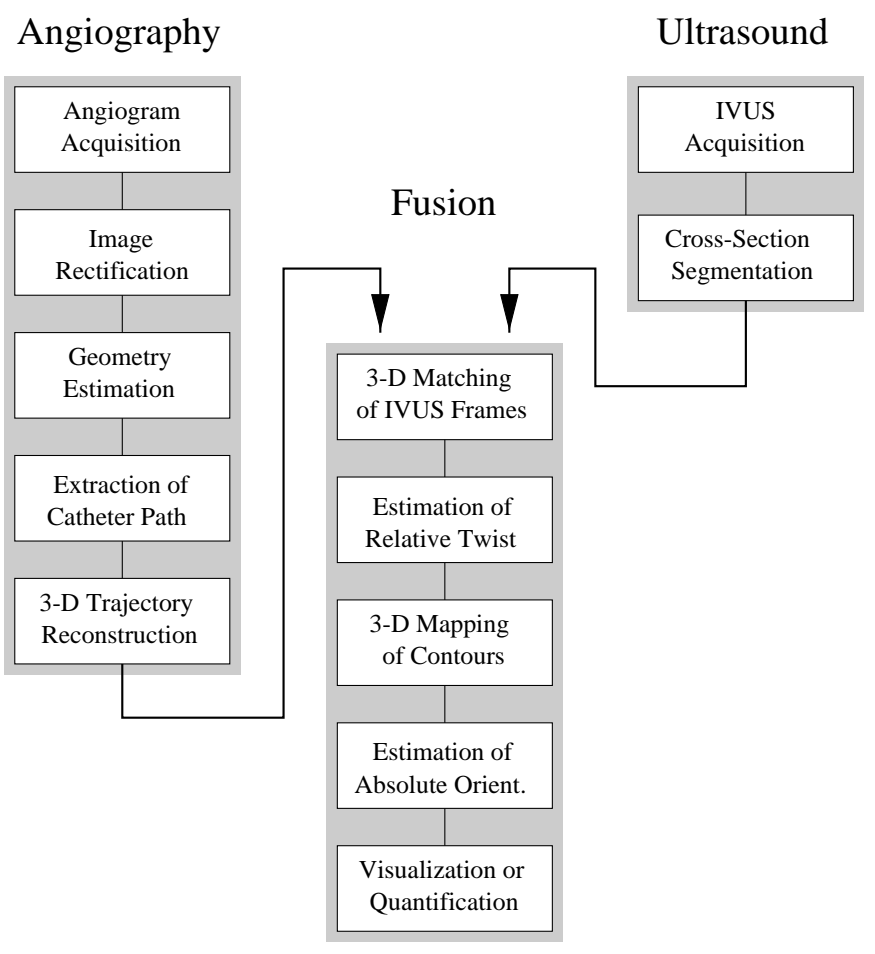

Fig. 5. Parallel processing of the angiograms and the IVUS images, followed by the steps of image fusion.
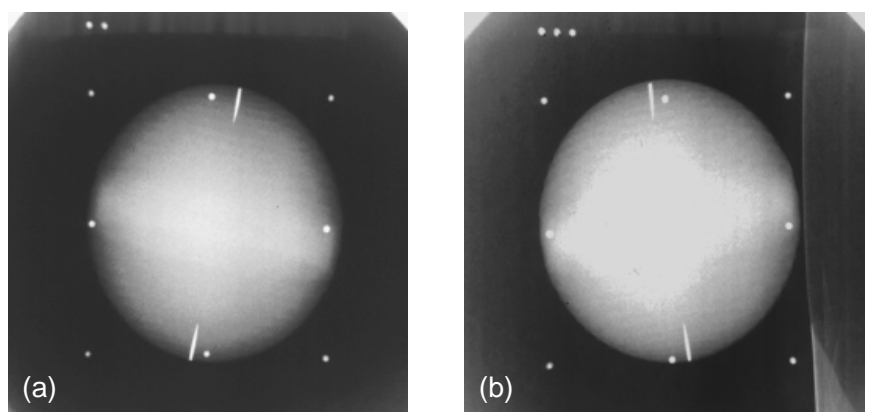

Fig. 6. Original angiograms of the calibration ball in (a) frontal and (b) lateral projection; after dewarping of the images using the eight markers, the nails at the poles of the ball may be used for biplane calibration.

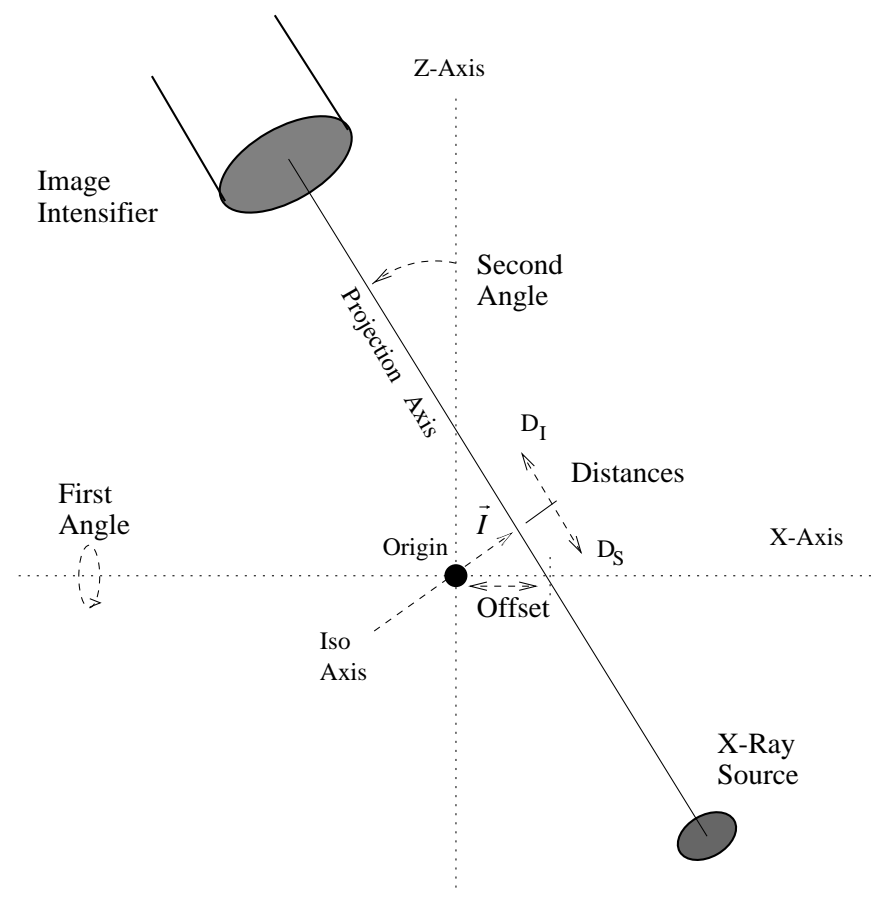

Fig. 7. The five degrees of freedom per X-ray system that have to be considered by the geometric model.

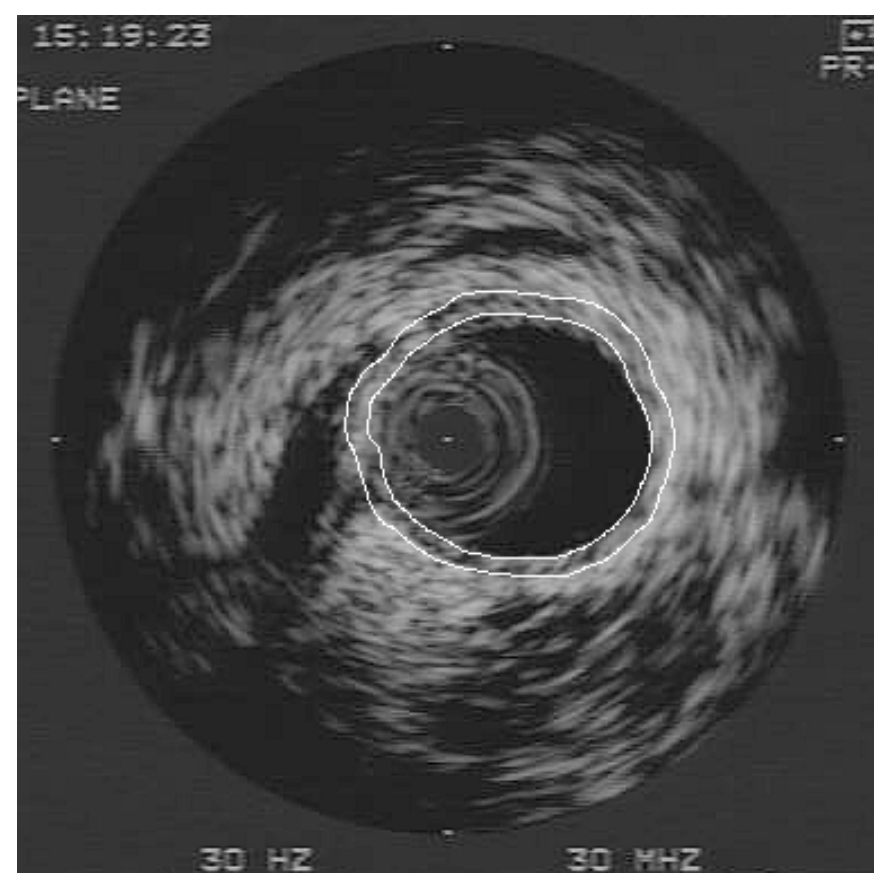

Fig. 8. Result of the border detection in IVUS images; the internal and external laminae have been correctly identified; plaque was not present in this example image. 


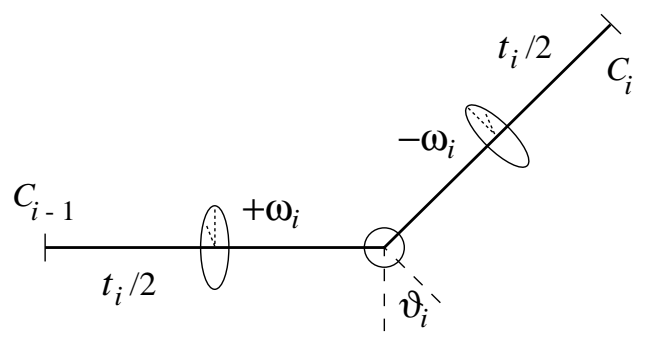

Fig. 9. Definition of the generalized torsion-free joint with three degrees of freedom.

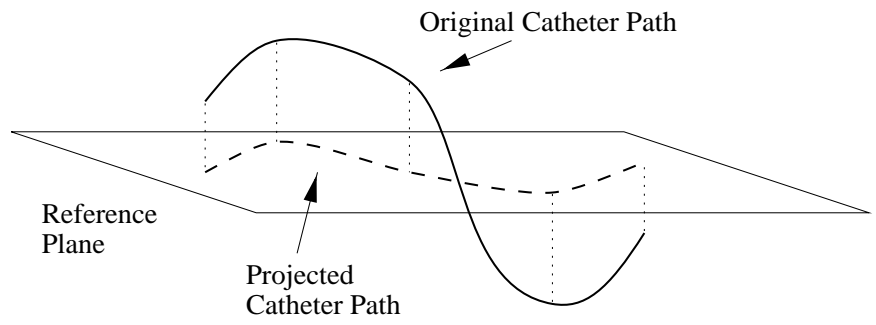

Fig. 11. Reference plane for generation of a torsion-free copy of the actual catheter path.

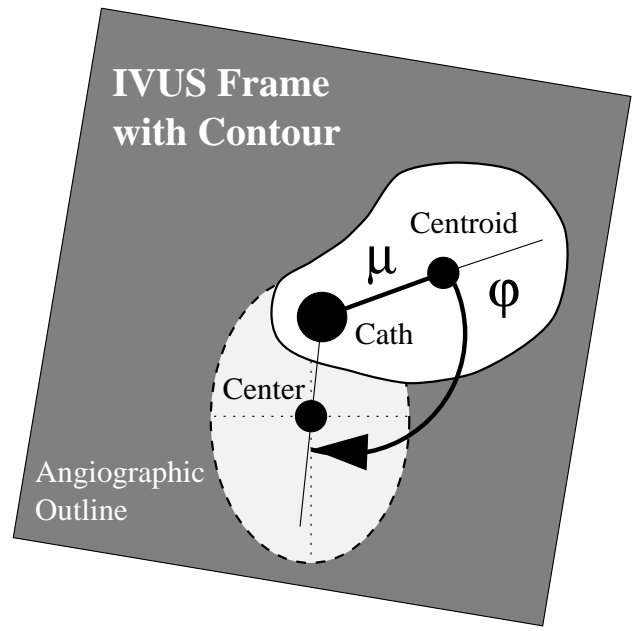

Fig. 12. Out-of-center strength $\mu$ and error angle $\varphi$ for estimation of the absolute orientation. (a)
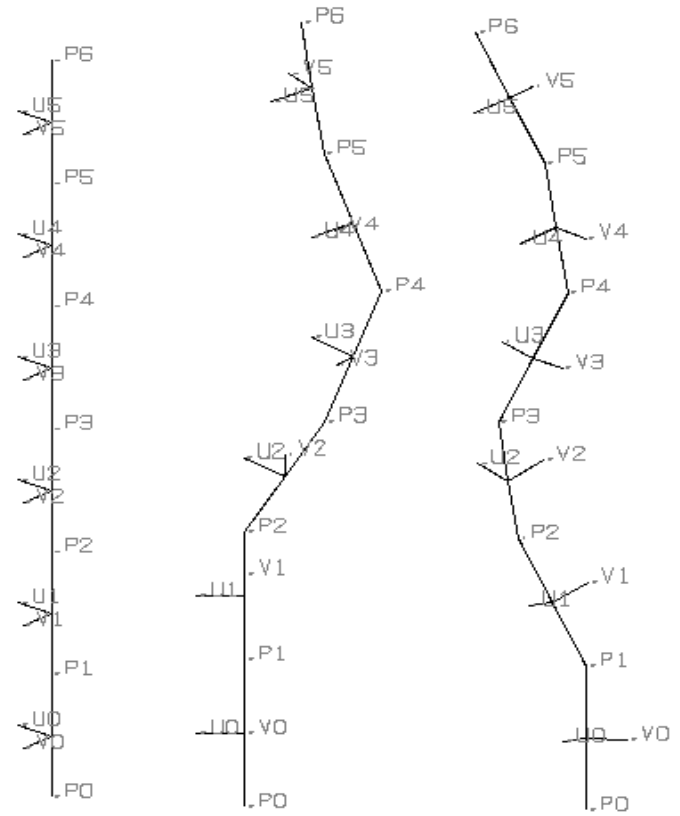

(b)
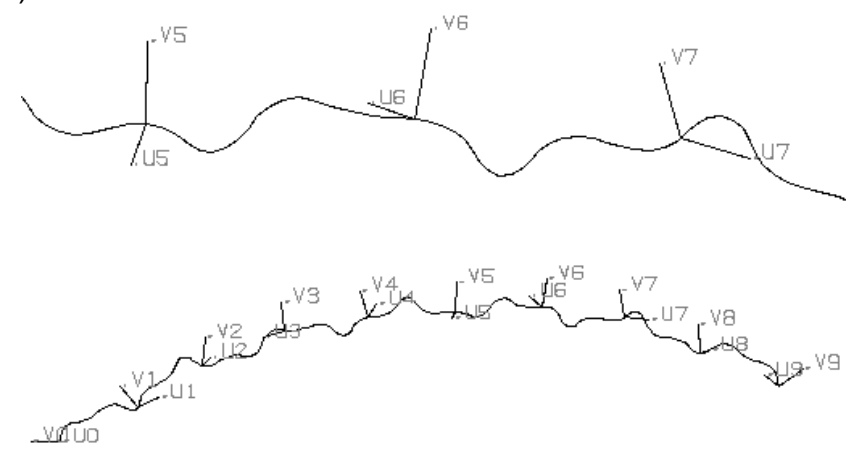

Fig. 13. Simulated catheter paths; (a) with five discrete joints, (b) with extreme curvature and torsion.

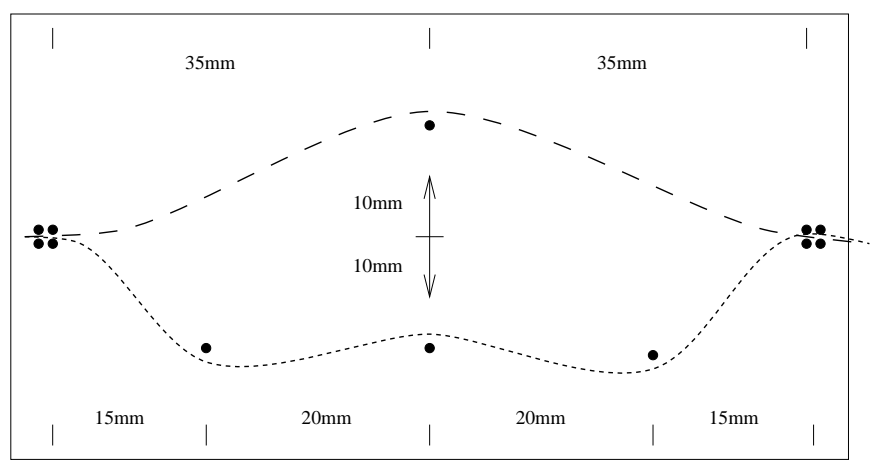

Fig. 14. Nail plate for verification of unsystematic twists. 


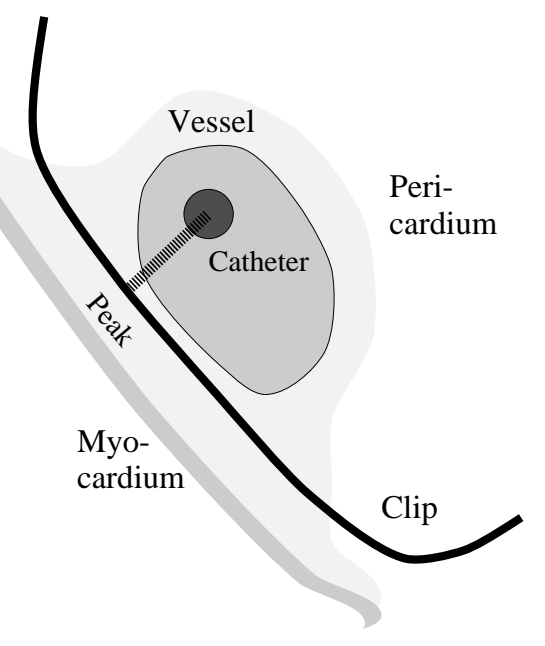

Fig. 15. Generation of the echo caused by a paper clip.

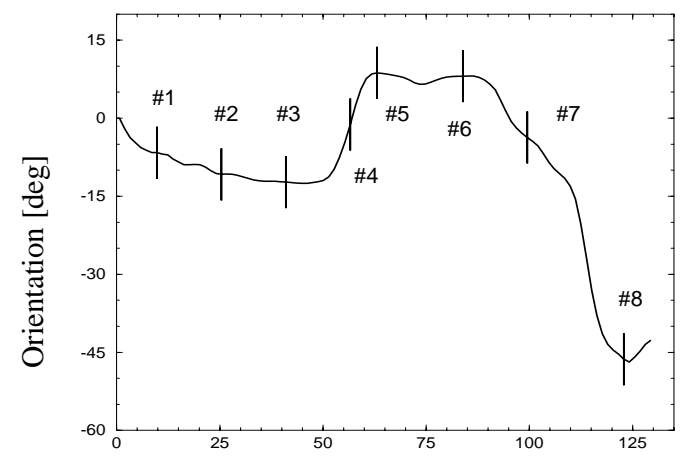

(a)

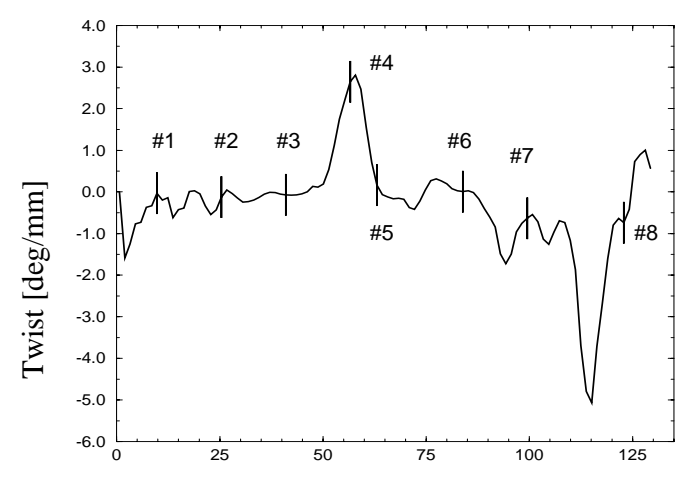

(b)

Fig. 18. Twisting behavior of the setup as shown in Fig. 16; (a) absolute axial orientation relative to the first IVUS frame, and (b) relative local twisting between adjacent IVUS frames.
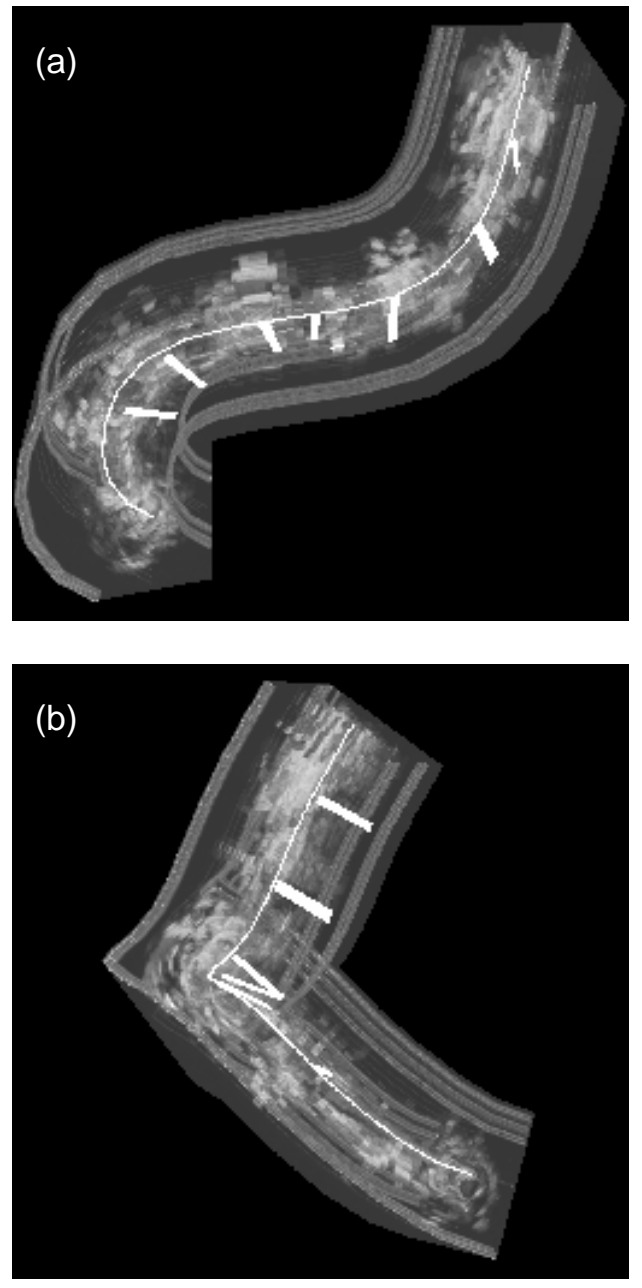

Fig. 19. Maximum intensity projections of the clips in (a) frontal and (b) lateral orientation, compare the reconstructions with the corresponding angiograms shown in Fig. 16a, b; the clip echoes have been enhanced in the IVUS images before reconstruction for better visibility. 


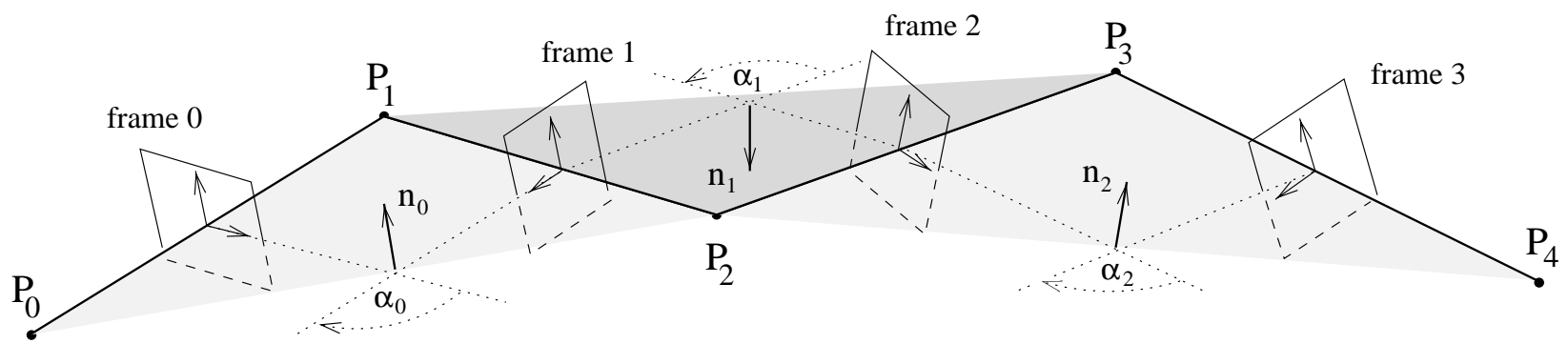

Fig. 10. Sequential triangulation method for estimation of the relative catheter twist.
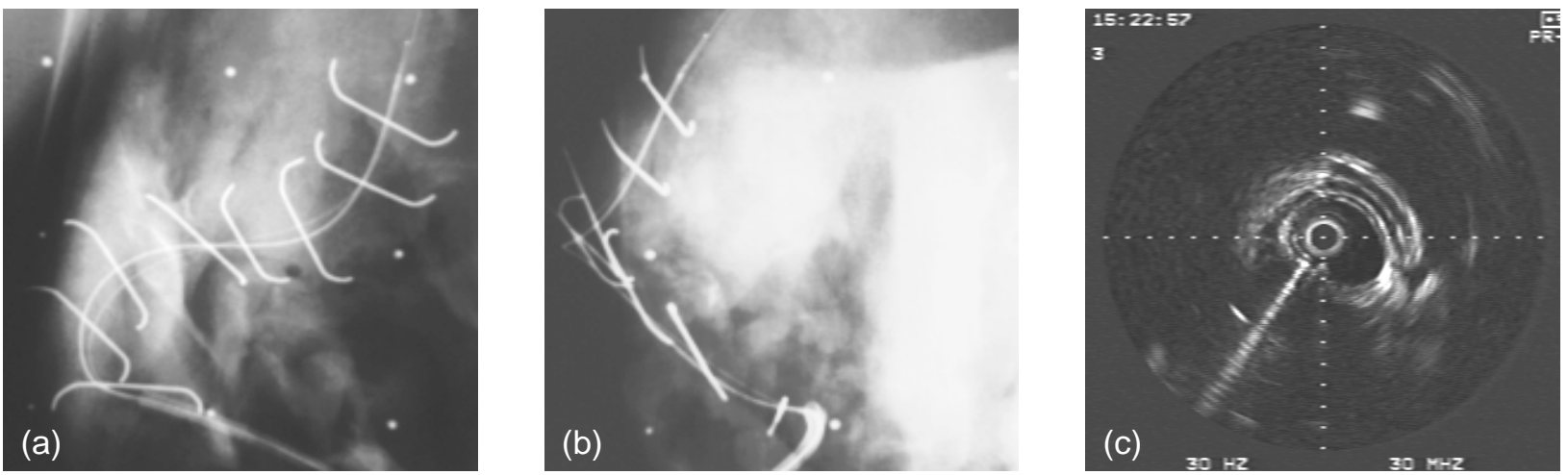

Fig. 16. Cadaveric pig heart with clips; (a) frontal and (b) lateral angiographic images, (c) IVUS image with peak.
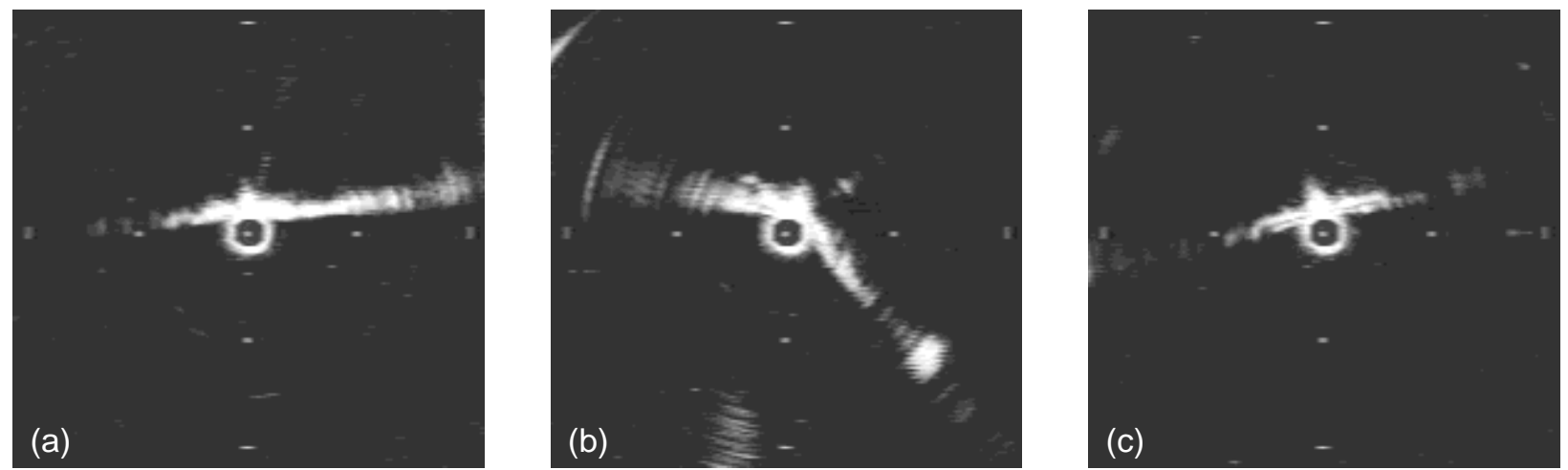

Fig. 17. Distortions due to bending; IVUS images (a) $8 \mathrm{~mm}$ distal from, (b) closest to, and (c) $8 \mathrm{~mm}$ proximal from an outer nail. 